\title{
CONVERGENCE OF SEQUENCES OF CONVEX SETS, CONES AND FUNCTIONS. II
}

\author{
BY \\ R. A. WIJSMAN(1)
}

0. Summary. A definition is given of convergence of a sequence of sets to a set, written $X_{n} \rightarrow X$, where $X$ and the $X_{n}$ are subsets of Euclidean $m$-space $E^{m}$. A new mode of convergence of a sequence of real valued functions on $E^{m}$ to a function is introduced, termed infimal convergence, and written $f_{n} \rightarrow$ inf $f$. $P(X), A(X), h(X)$ and $X^{*}$ are the projecting cone, asymptotic cone, support function and polar, respectively, of $X .[X, f]=\{(x, a): x \in X, a \geqq f(x)\}$, where $X=\{x: f(x)<\infty\}$, and $L_{a}(f)=\{x: f(x) \leqq a\}$. In the statements of the theorems below it is understood that all sets are convex, limit sets are nonempty, and all functions are convex and closed (= lower semicontinuous). Theorem 3.2 states that if $X_{n} \rightarrow X$ and $C$ is any open cone covering $A(X)$ then there exist $N$ and a disk $D(R)=\{x:|x| \leqq R\}$ such that $X_{n} \subset C \cup D(R)$ for $n>N$. Theorem 4.1 proves that if $X_{n} \rightarrow X$ and the origin of $E^{m}$ is not in $X$, then $P\left(X_{n}\right) \rightarrow P(X)$. Two more proofs of Theorem 4.1 are given, one using support functions (in \$6), the other using support functions, level sets and polars (in §7). Theorems 5.1 and 6.3 together show that $X_{n} \rightarrow X$ if and only if $h\left(X_{n}\right) \rightarrow_{\text {inf }} h(X)$. Theorem 6.1 states that $\left[X_{n}, f_{n}\right] \rightarrow[X, f]$ if and only if $f_{n} \rightarrow$ inf $f$, and Theorem 6.2 that $f_{n} \rightarrow$ inf $f$ if and only if $\phi_{n} \rightarrow$ inf $\phi$, where $\phi$ is the conjugate function of $f, \phi_{n}$ of $f_{n}$. In Theorem 7.1 it is shown that $f_{n} \rightarrow$ inf $f$ implies $L_{a}\left(f_{n}\right) \rightarrow L_{a}(f)$ provided $a \neq \inf f$. Theorem 7.2 shows that $X_{n} \rightarrow X$ implies $X_{n}^{*} \rightarrow X^{*}$. Several examples are given to show that the theorems are false without the various conditions made, such as convexity. Examples are also given to show that in general pointwise and infimal convergence of functions are incomparable.

1. Introduction. The present study on sequences of convex sets was motivated by the following question that arose in a proof of the optimum property of sequential probability ratio tests [1]: Given a closed, convex set $X$ and a sequence of closed, convex sets $X_{n}$ in the plane; let $x$ be a point that is in none of the $X_{n}$, nor in $X$, and consider the two supporting lines through $x$ of $X_{n}$, and of $X$ : then if $X_{n}$ converges to $X$ in some reasonable sense (to be made precise later), is the same true for the corresponding supporting lines $[1, \S \S 4$ and 5$]$ ? This question can

Received by the editors November $1,1963$.

(1) Work supported by the National Science Foundation, grant NSF G-21507. 
be generalized in more than two dimensions by replacing the supporting lines of $X$ through $x$ by the projecting cone of $X$ with vertex in $x$, and similarly ifor the $X_{n}$. Before this question can be answered, a satisfactory definition of convergence of $X_{n}$ to $X$ has to be given. For closed, bounded sets this can be done in terms of the Hausdorff metric (cf. e.g. [5]). However, in applications the sets $X_{n}$ are often unbounded (as in [1]), and in any case the projecting cones are unbounded. Although it is possible to generalize the Hausdorff metric to unbounded sets, it is simpler to define convergence in terms of the distance function. This is done in $\$ 3$. The question of the convergence of projecting of cones is answered in the affirmative in Theorem 4.1.

When studying convex sets it comes natural to study the corresponding support functions. This is done in $\S 5$. It seems plausible that a sequence of convex sets converges to a set if and only if this is true for the corresponding support functions. However, this is not so is pointwise convergence of a sequence of functions is considered. We can achieve the desired equivalence only by introducing a new type of convergence of a sequence of functions, which we shall term infimal convergence. Thus, if all the sets are convex, a sequence of sets converges to a set if and only if for the corresponding support functions we have infimal convergence.

The concept of infimal convergence has proved itself useful throughout the the present study. For instance, it is shown in $\$ 6$ that a sequence of convex and closed (to be defined in \$2) functions converges infimally to a function if and only if the same is true for the corresponding conjugate functions. This provides a second proof of Theorem 4.1. In $\$ 7$ we consider the level sets of functions, and the polars of sets. It is shown that if a sequence of convex and closed functions converges infimally to a function, the same is true for the corresponding level sets, under a mild condition. In the same section it is proved that if a sequence of convex sets converges to a set, the same is true for their polars. This provides a third proof of Theorem 4.1.

Unless a more general space is specified, all sets are subsets of some finite dimensional Euclidean space. However, most theorems are valid in more general topological spaces. Furthermore, although for simplicity we shall phrase definitions and theorems in terms of sequences, we may replace "sequence" by "net" throughout. An announcement of the main results in this paper was made in [6].

2. Notation and definitions. The complement of a set $X$ is denoted $X^{\sim}$, the closure of $X$ by $X$ or $C 1 X$. Euclidean $m$-space is denoted by $E^{m}$, and its origin by 0 . If $x \neq 0$, a ray from 0 through $x$ is denoted $(x)$, following Fenchel [3]. Let $d(x, y)$ be the Euclidean distance between $x$ and $y$, which will also be written as $|x-y|$, where || denotes Euclidean norm. The inner product of $x$ and $y$ is written $x \cdot y$. In $E^{m}$ let $D(R)=\{x:|x| \leqq R\}$ be the $m$-disk centered at 0 and of radius $R, 0<R<\infty$. For any set $X$ we shall for short denote $X \cap D(R)$ by $X^{R}$. 
In the space of rays we can introduce a metric by defining the distance between $(x)$ and $(y)$ to be $d(x /|x|, y /|y|)$ (this amounts to assigning to each ray $(x)$ the point $x /|x|$ on the unit $(m-1)$-sphere, and taking the chord distance topology on the latter). A cone is a union of rays. We shall consider a cone $C$ sometimes as a point set in $E^{m}$, and sometimes as a point set in the space of rays, without changing the notation. However, with an open (or closed) cone is always meant cone open (or closed) in the metric topology of rays. We shall also use the symbol $d$ for distance in the space of rays: $d((x),(y)), d(C,(x))$, etc. The projecting cone of a set $X$ is defined as $P(X)=\{(x): x \in X\})$. The asymptotic cone of $X$ is $A(X)=\left\{(x):(x)=\lim \left(x_{n}\right), x_{n} \in X,\left|X_{n}\right| \rightarrow \infty\right\}$. Obviously, bounded sets have empty asymptotic cones. Properties of projecting and asymptotic cones can be found in [3].

The distance of a point $x$ to a nonempty set $X$, denoted $d(X, x)$, is the usual distance inf $\{|x-y|: y \in X\}$. With the distance function $d(X)$ of $X$ is meant $d(X, \cdot)$. If $X$ is empty, we shall define $d(X)$ to be identically $+\infty$. The support function $h(X)$ of a nonempty set $X$ is defined by its value $h(X, \xi)$ at any point $\xi: h(X, \xi)=\sup _{x \in X} \xi \cdot x$. Properties of the support function can be found for instance in [2].

All functions are understood to be real valued, with $+\infty$ values allowed. If $\left\{f_{n}\right\}$ converges to $f$ pointwise, we shall write as usual $f_{n} \rightarrow f$. When the symbols lim, lim sup or lim inf are used, it will always be understood that these limits are taken as $n \rightarrow \infty, n$ running over the integers. If $f$ is a function on $E^{n}$ and $\rho>0$, we define the function ${ }_{\rho} f$ on $E^{m}$ by ${ }_{\rho} f(x)=\inf \{f(y):|y-x|<\rho\}$. A function $f$ is lower semicontinuous if and only if $\lim _{\rho \rightarrow 0} f=f$. For convenience, following Fenchel [3], we shall call such a function closed in the following (there is a close connection between closed, convex functions and closed, convex sets, see $\S \S 6$ and 7). (In [3] the definition is given only for convex functions, is different from the above definition, but equivalent to it for convex functions.)

DEFinition. We shall say that a sequence $\left\{f_{n}\right\}$ of functions converges infimally to $f$, written $f_{n} \rightarrow$ inf $f$, if

$$
\lim _{\rho \rightarrow 0} \liminf f_{n}=\lim _{\rho \rightarrow 0} \lim \sup _{\rho} f_{n}=f
$$

Other concepts will be defined as the need arises.

3. Convergence of sequences of sets. As an example consider the following sequence of circles in the $(x, y)$-plane: $X_{n}=\left\{(x, y): x^{2}+y^{2}-2 n y=0\right\}$. As $n \rightarrow \infty$ the sequence converges in an intuitive sense to the $x$-axis $X=\{(x, y): y=0\}$. For bounded sets the intuitive notion of convergence of sets can be made precise by introducing the Hausdorff distance between sets (see e.g. [5]; the distance between $X$ and $Y$ can be defined simply in terms of their distance functions as $\left.\max _{x}|d(X, x)-d(Y, x)|\right)$. This does no longer work for unbounded sets. 
Instead, we shall define convergence directly, instead of in terms of a metric, as follows:

Definition 3.1. We shall say that the sequence $\left\{X_{n}\right\}$ converges to the set $X$, written $X_{n} \rightarrow X$, if $d\left(X_{n}\right) \rightarrow d(X)$.

It can be verified immediately that according to the above definition the sequence of circles in our example converges indeed to the $x$-axis. It is easily shown that if $X_{n} \rightarrow X$ and the $X_{n}$ are convex, then $X$ is convex. For closed limit sets Definition 3.1 corresponds to a definition given by Frolík [4] for general topological spaces $\left({ }^{2}\right)$. Note that $d(X)=d(X)$, so that limits of convergent sequences are unique only up to closures. Since for any nonempty set $X$ and points $x, y$ we have

$$
|d(X, x)-d(X, y)| \leqq|x-y|,
$$

every family of distance functions satisfies a uniform Lipschitz condition. Then by Ascoli's theorem, if $d\left(X_{n}\right) \rightarrow d(X)$, and $X$ nonempty, the convergence is uniform on every compact set. It is of some interest, although we shall not use it, that it is possible to define a pseudo distance between arbitrary sets $X$ and $Y$ with which Definition 3.1 is in agreement: it is the value of $\varepsilon$ such that

$$
\max _{|x| \leqq 1 / \varepsilon}|d(X, x)-d(Y, x)|=\varepsilon .
$$

This defines a complete metric if the sets are closed.

The following theorem is stated for a somewhat more general metric space than $E^{m}$, since it will be applied to the metric space of rays in Theorem 4.1.

THEOREM 3.1. In a metric space where every disk is compact, $X_{n} \rightarrow X$ if and only if $d\left(X_{n}, x\right) \rightarrow 0$ for every $x \in X$ and $\lim \inf d\left(X_{n}, x\right)>0$ for every $x \notin \bar{X}$.

Proof. It is sufficient to prove the theorem for $X$ closed. The "only if" part is obvious. To prove the "if" part we have to show $d\left(X_{n}, x\right) \rightarrow d(X, x)$ for every $x$. This is certainly true for $x \in X$, since then $d(X, x)=0$ and $d\left(X_{n}, x\right) \rightarrow 0$ by hypothesis. Suppose then $x_{0} \notin X$ so that $d\left(X, x_{0}\right)=r>0$. We distinguish between two cases: $X$ nonempty and $X$ empty. If $X$ is nonempty, let $x_{1} \in X$ be such that $\left|x_{0}-x_{1}\right|=r$. Since $d\left(X_{n}, x_{1}\right) \rightarrow 0$ and

$$
d\left(X_{n}, x_{0}\right) \leqq d\left(X_{n}, x_{1}\right)+\left|x_{0}-x_{1}\right|,
$$

by (3.1), we have $\lim \sup d\left(X_{n}, x_{0}\right) \leqq r$. Whe shall show now $\lim \inf d\left(X_{n}, x_{0}\right) \geqq r$ so that then $\lim d\left(X_{n}, x_{0}\right)=r=d\left(X, x_{0}\right)$. Choose any $\varepsilon$ such that $0<\varepsilon<r$, and let $D$ be the disk about $x_{0}$ of radius $r-\varepsilon$, so that $D$ and $X$ are disjoint. By hypothesis, in each point $x \in D \lim \inf d\left(X_{n}, x\right)>0$, so that there is an integer $n(x)$ and a spherical neighborhood $U(x)$ such that $U(x)$ does not meet $X_{n}$ for any $n>n(x)$. Since $D$ is compact, it can be covered by a finite number of these neighborhoods. Let $N$ be the maximum of the corresponding $n(x)$ 's, then $D$ does

(2) I am indebted to L. L. Helms for bringing this reference to my attention. 
not meet $X_{n}$ for $n>N$. Consequently, $d\left(X_{n}, r_{0}\right) \geqq r-\varepsilon$ for all $n>N$ so that $\lim \inf d\left(X_{n}, x_{0}\right) \geqq r-\varepsilon$. Since $\varepsilon$ is arbitrary, the conclusion follows. In case $X$ is empty we may choose $r$ arbitrarily large, and, using the same disk $D$ as before, we conclude $\lim \inf d\left(X_{n}, x_{0}\right) \geqq r$, so that $d\left(X_{n}, x_{0}\right) \rightarrow \infty=d\left(X, x_{0}\right)$.

The following lemma states, roughly speaking, that all but a bounded portion of a set is contained in its asymptotic cone.

LEMma 3.1. Let $X$ be $a$ set and $C$ an open cone such that $C \supset A(X)$. Then there exists $R$ such that $X \subset C \cup D(R)$.

Proof. Suppose the lemma were false, then there is a sequence

$$
\left\{x_{n}\right\}, \quad x_{n} \in X, x_{n} \notin C,\left|x_{n}\right| \rightarrow \infty .
$$

In the space of rays the sequence $\left\{\left(x_{n}\right)\right\}$ lies in the compact set $C^{\sim}$ so that there is a subsequence converging to a ray $(x) \in C^{\sim}$. On the other hand, $(x) \in A(X)$ by the definition of $A(X)$. This is a contradiction since $A(X)$ and $C^{\sim}$ are disjoint.

Corollary 3.1. If $C_{1}$ and $C_{2}$ are open cones such that $\bar{C}_{1} \subset C_{2}$, then for every $a \in E^{m}$ there is $R$ such that $C_{1}+a \subset C_{2} \cup D(R)$.

Proof. We apply Lemma 3.1 with $X=C_{1}+a, C=C_{2}$, observing that $A\left(C_{1}+a\right)=A\left(C_{1}\right)=\bar{C}_{1}$.

So far we have not invoked convexity. The next theorem is the first one where convexity is essential. It says, roughly, that if a sequence of convex sets converges to $X$, then they all are eventually contained in $A(X)$ except possibly for a bounded region. A counterexample for nonconvex sets is the example of the sequence of circles in the beginning of this section.

THEOREM 3.2. Let $\left\{X_{n}\right\}$ be a sequence of convex sets, and $X$ a nonempty convex set such that $X_{n} \rightarrow X$. Then for every open cone $C \supset A(X)$ there exist $R$ and $N$ such that $X_{n} \subset C \cup D(R)$ for all $n>N$.

Proof. Without loss of generality we may choose the origin 0 at our pleaure. For, suppose the theorem were false for 0 but true for $0^{\prime}=0+a$. There exists then an open cone $C_{2} \supset A(X)$ and a sequence $\left\{x_{n}\right\}$ with $x_{n} \in X_{n}, x_{n} \notin C_{2},\left|x_{n}\right| \rightarrow \infty$. Choose an open cone $C_{1}$ such that $A(X) \subset C_{1} \subset \bar{C}_{1} \subset C_{2}$. Since $\left|x_{n}\right| \rightarrow \infty$ and since the asymptotic cone of $X$ for the origin $0^{\prime}$ is $A(X)+a$, which is $\subset C_{1}+a$, applying the theorem with origin $0^{\prime}$ we must have that $x_{n}$ is eventually in $C_{1}+a$. Then according to Corollary $3.1 x_{n}$ is eventually in $C_{2}$, but this contradicts $x_{n} \notin C_{2}$.

Choose as origin 0 any point of $X$. Observe that $A(X)=\{a x: a \geqq 0$, $\left.x=\lim x_{n}|| x_{n}\left|, x_{n} \in X,\right| \exists x_{n} \mid \rightarrow \infty\right\}$. Suppose the theorem false, then there would exist a sequence $\left\{x_{n}\right\}, x_{n} \in X_{n}, x_{n} \notin C,\left|x_{n}\right| \rightarrow \infty$. Put $y_{n}=x_{n}|| x_{n} \mid$, then $\left|y_{n}\right|=1$ so that we may assume $\lim y_{n}$ exists, say $y_{n} \rightarrow x$, where $x \neq 0$ since $\left|y_{n}\right|=1$. Since $X_{n} \rightarrow X$ and $0 \in X$, there is a sequence $\left\{s_{n}\right\}, s_{n} \in X_{n}, s_{n} \rightarrow 0$. Choose 
$a>0$ arbitrary and put $t_{n}=\left(1-a /\left|x_{n}\right|\right) s_{n}+\left(a /\left|x_{n}\right|\right) x_{n}$ then eventually $t_{n} \in X_{n}$ since $X_{n}$ is convex. Furthermore, $t_{n} \rightarrow a x$ and therefore $a x \in X$ because $X_{n} \rightarrow X$. Since this holds for all $a>0, x \in A(X)$. On the other hand, since $x_{n} \notin C, y_{n} \notin C$ so that $x=\lim y_{n} \in \mathrm{Cl} C^{\sim}=C^{\sim} \cup\{0\}$. But $x \neq 0$, so that $x \in C^{\sim}$. This contradicts $x \in A(X)$.

It is easy to see that the theorem is false without the condition that $X$ be nonempty. For example, on the real line take $X_{n}$ to consist of the single point $n$.

4. Projecting cones. If $X_{n} \rightarrow X$, is it true that $P\left(X_{n}\right) \rightarrow P(X)$ ? That this is not so in general is easily seen from the example in the beginning of $\S 3$. There $P\left(X_{n}\right)$ is the upper half plane for each $n$, whereas $P(X)=X$ is the $x$-axis. The trouble seems to be that the $X_{n}$ are not convex. If we modify the example by letting $X_{n}=\left\{(x, y): x^{2}+y^{2}-2 n y \leqq 0\right\}$ and $X=\{(x, y): y \geqq 0\}$ then the sets are convex and $P\left(X_{n}\right) \rightarrow P(X)$. However, if we modify the example once more by letting $X_{n}=\left\{(x, y): x^{2}+(y-n+(1 / n))^{2} \leqq n^{2}\right\}$ and $X$ as before, then the sets are still convex, $X_{n} \rightarrow X$, but $P\left(X_{n}\right)$ is the whole plane for each $n$ whereas $P(X)=X$ is the upper half plane. The trouble in this example is that 0 is a point of the limit set $X$. Even if 0 is not in $X$ but in its closure, a counterexample is easily constructed: take the $X_{n}$ as in the previous example but let $X$ now be the open upper half plane. These counterexamples justify the choice of the hypotheses in Theorem 4.1 below. A second proof of Theorem 4.1 will appear after Theorem 6.3, using support functions, and a third proof after Theorem 7.2, using level sets and polars.

THEOREM 4.1. Let $\left\{X_{n}\right\}$ be a sequence of convex sets and $X$ a nonempty set such that $0 \notin \bar{X}$ and $X_{n} \rightarrow X$. Then $P\left(X_{n}\right) \rightarrow P(X)$.

First Proof $\left({ }^{3}\right)$. The definition $X_{n} \rightarrow X$ is equivalent to: $\lim \inf X_{n}=\lim \sup X_{n}=\bar{X}$, where we define $\lim \inf X_{n}\left(\lim \sup X_{n}\right)$ to be the set of points $x$ such that for every neighborhood $U$ of $x, U \cap X_{n}$ is eventually not empty (is not empty for infinitely many $n$ ). Both $\lim \inf X_{n}$ and $\lim \sup X_{n}$ are closed. The theorem will be proved after showing (1) $\mathrm{Cl} P(X) \subset \lim \inf P\left(X_{n}\right)$, and (2) lim sup $P\left(X_{n}\right) \subset \mathrm{Cl} P(X)$.

(1) Let $\alpha x \in P(X), \alpha \geqq 0, x \in X$. Since $X_{n} \rightarrow X$, there exists $\left\{x_{n}\right\}, x_{n} \in X_{n}$, $x_{n} \rightarrow x$. Then $\alpha x_{n} \in P\left(X_{n}\right)$ and $\alpha x_{n} \rightarrow \alpha x$, so $\alpha x \in \lim$ inf $P\left(X_{n}\right)$ which shows $P(X) \subset \lim \inf P\left(X_{n}\right)$, so $\mathrm{Cl} P(X) \subset \lim \inf P\left(X_{n}\right)$.

(2) Let $p \in \lim \sup P\left(X_{n}\right)$. By taking a suitable subsequence of $\{n\}$, which, however, will again be denoted $\{n\}$, there exists $\left\{p_{n}\right\}, p_{n} \in P\left(X_{n}\right), p_{n} \rightarrow p$. Now $p_{n}=\alpha_{n} x_{n}$ for some $\alpha_{n} \geqq 0, x_{n} \in X_{n}$, so $\alpha_{n} x_{n} \rightarrow p$. We cannot have $\alpha_{n} \rightarrow \infty$ for then $x_{n} \rightarrow 0$ which is excluded by $X_{n} \rightarrow X$ and $0 \notin X$. Therefore, $\lim \inf \alpha_{n}<\infty$ and, by taking a subsequence, we may assume $\alpha_{n} \rightarrow \alpha<\infty$. If $\alpha>0, x_{n} \rightarrow p / \alpha$ so

(3) This proof was outlined by Victor Klee in his review of [6], Math. Reviews 28 (1964), no. 514. I am very indebted to Professor Klee for his elegant proof, which replaces my longer original proof. The same ideas made it also possible to shorten the proof of Theorem 3.2. 
that $p / \alpha \in \bar{X}$, hence $p \in P(\bar{X}) \subset \mathrm{Cl} P(X)$. If $\alpha=0, x_{n} \rightarrow \infty$. Take any $z \in X$ and $\left\{z_{n}\right\}$, $z_{n} \in X_{n}, \quad z_{n} \rightarrow z$. Take $a$ arbitrary, $0<a<\infty$, and put $y_{n}=\left(1-a \alpha_{n}\right) z_{n}+a \alpha_{n} x_{n}$, then $y_{n} \in X_{n}$ eventually, by convexity. Now $y_{n} \rightarrow z+a p$ so that $z+a p \in X$ and therefore $p+z / a \in P(\bar{X}) \subset \mathrm{Cl} P(X)$. By letting $a \rightarrow \infty$ we find $p \in \mathrm{Cl} P(X)$, which shows lim sup $P\left(X_{n}\right) \subset \mathrm{Cl} P(X)$.

Using the example at the end of $\S 3$ it is easily seen that Theorem 4.1 is false without the condition that $X$ be nonempty.

5. Support functions. The support function of a nonempty set was defined in $\$ 2$. It is of some interest to note that a support function is always closed (see the definition of a closed function in \$2) since it is the supremum of a family of continuous functions. Since the convex hull of a set has the same support function as the set itself, the support function does not determine the set uniquely, not even up to closure. If only closed, convex sets are considered, then there is a one to one correspondence between sets and support functions. Therefore, one is tempted to conjecture that if the $X_{n}$ are convex, then $X_{n} \rightarrow X$ if and only if $h\left(X_{n}\right) \rightarrow h(X)$. However, this turns out to be false in both directions, as shown by the following two examples.

EXAmPLE 5.1. In the $(x, y)$-plane let $X_{n}=\{(x, y): n x+y \leqq 0\}$, then $X_{n} \rightarrow X=\{(x, y): x \leqq 0\}$. Taking $\xi=(1,0)$, we have $h(X, \xi)=0$, but for every $n=1,2, \cdots, h\left(X_{n}, \xi\right)=\infty$.

EXAMPLE 5.2. In the $(x, y)$-plane let $X_{n}=\{(x, y): x \leqq 0$ and $y-n x \geqq n\}$, and $X=\{(x, y): x \leqq 0\}$. Then $X_{n} \rightarrow X^{\prime}=\{(x, y): x \leqq-1\} \not \equiv X$. On the other hand, writing $\xi=\left(\xi_{1}, \xi_{2}\right)$, we have $h(X, \xi)=0$ if $\xi_{1} \geqq 0$ and $\xi_{2}=0, h(X, \xi)=\infty$ otherwise, and $h\left(X_{n}, \xi\right)=n \xi_{2}$ if $\xi_{1} \geqq 0$ and $0 \leqq-n \xi_{2} \leqq \xi_{1}, h\left(X_{n}, \xi\right)=\infty$ otherwise. It can readily be verified that $h\left(X_{n}, \xi\right) \rightarrow h(X, \xi)$ for every $\xi$.

In order to get equivalence between convergence of convex sets and convergence of their support functions we need a sense of convergence of functions different from pointwise. It turns out that what is needed is precisely the notion of infimal convergence as defined in $\$ 2$. It is easily verified that in Example 5.1 $\left\{h\left(X_{n}\right)\right\}$ converges infimally to $h(X)$ and in Example 5.2 it does not (this also follows from Theorem 5.1 below). Thus, pointwise and infimal convergence are not comparable in general, i.e. neither implies the other, not even for support functions, which have the nice properties of being convex, closed, and positively homogeneous. However, it does happen often that a sequence of functions converges in both senses, i.e. $f_{n} \rightarrow_{\text {inf }} f$ and $f_{n} \rightarrow f^{\prime}$, and in that case we have necessarily $f \leqq f^{\prime}$.

We shall first prove a few lemmas, of which only Lemma 5.2 is used in this section. Lemmas 5.1 and 5.3 will not be used until $\$ 6$, and Corollary 5.1 not until \$7. Note that the support function of an empty set is undefined. Yet, we shall often write $h\left(X_{n}\right)$ without demanding $X_{n}$ to be nonempty for all $n$. However, if $X_{n} \rightarrow X, X$ nonempty, then $X_{n}$ is nonempty for $n>N_{0}$, for some $N_{0}$. In the 
remainder of the paper we shall always understand $n>N_{0}$ without mentioning this explicitly.

LEMm 4 5.1. Let $\left\{f_{n}\right\}$ be a sequence of functions and define $f^{*}=\lim _{\rho \rightarrow 0}$ $\limsup f_{n}, f_{*}=\lim _{\rho \rightarrow 0} \liminf _{\rho} f_{n}$. Then for every $\rho>0$

$$
\lim \sup _{\rho} f_{n} \leqq f^{*}
$$

and a similar inequality with lim sup replaced by $\lim \inf$ and $f^{*}$ by $f_{*}$. Furthermore, $f^{*}$ and $f_{*}$ are closed.

Proof. Choose $x, \rho>0$ and $\varepsilon>0$ arbitrarily. There exists $y$ with $|y-x|<\rho$ such that $f^{*}(y)<{ }_{\rho} f^{*}(x)+\varepsilon$. Since by hypothesis $\lim _{\delta \rightarrow 0} \lim \sup _{\delta} f_{n}(y)=f^{*}(y)$, we have for every $\delta>0, \lim _{\sup _{\delta}} f_{n}(y) \leqq f^{*}(y)$. Take $\delta$ such that $0<\delta<\rho-|y-x|$ then clearly ${ }_{\rho} f_{n}(x) \leqq{ }_{\delta} f_{n}(y)$, so that $\lim \sup _{\rho} f_{n}(x) \leqq \lim \sup _{\delta} f_{n}(y) \leqq f^{*}(y)$ $<{ }_{\rho}^{*}(x)+\varepsilon$. Letting $\varepsilon \rightarrow 0$ proves (5.1). The analogous inequality with lim inf and $f_{*}$ is proved in the same way. To show that $f^{*}$ is closed, take $\lim _{\rho \rightarrow 0}$ on both sides of (5.1), then we get $f^{*} \leqq \lim _{\rho \rightarrow 0 \rho} f^{*}$. But for every $\rho, f^{*} \geqq{ }_{\rho}^{*}$, so that $f^{*} \geqq \lim _{\rho \rightarrow 0 \rho} f^{*}$. Hence we have equality. The proof for $f_{*}$ is exactly the same.

Corollary 5.1. If $f_{n} \rightarrow_{\text {inf }} f$ then $f$ is closed.

Proof. In Lemma $5.1 f^{*}=f_{*}=f$.

LEMMA 5.2. If $\left\{X_{n}\right\}$ is a sequence of convex sets converging to a nonempty set $X$, and if $\xi$ is such that $h(X, \xi)=0$, the for every $\rho>0 \lim \sup _{\rho} h\left(X_{n}, \xi\right) \leqq 0$.

Proof. We may suppose in the proof that the $X_{n}$ and $X$ are closed. The theorem is obviously true for $\xi=0$. To prove the theorem for $\xi \neq 0$ we may suppose $|\xi|=1$, using the positive homogeneity of support functions. Let $\xi$, with $|\xi|=1$, be fixed in the following. For any $\eta \neq 0$ denote by $H_{\eta}$ the half space $\{x: \eta \cdot x \leqq 0\}$. Then $h(X, \xi)=0$ implies $X \subset H_{\xi}$, which, in turn, implies $A(X) \subset H_{\xi}$. For any $\varepsilon>: 0$, the open cone $C=\{(x): \xi \cdot x<\varepsilon|x|\}$ contains $H_{\xi}$. Therefore $C \supset A(X)$. It can be verified readily that there is a constant $c>0\left(c=2^{1 / 2}\right.$ will do $)$ such that $|\eta|=1$ and $H_{\eta} \subset C$ together imply $|\eta-\xi|<c \varepsilon$. Let $\rho>0$ be given and choose $\varepsilon=\rho / c$, then any $\eta$ with the above mentioned properties satisfies $|\eta-\xi|<\rho$. By Theorem 3.2 there exist $R$ and $N$ such that $X_{n} \subset C \cup D(R)$ for all $n>N$. For any $\delta>0$ consider the cone $C+\delta \xi$ with vertex in $\delta \xi$. Since $C+\delta \xi \supset C$, $X_{n} \subset(C+\delta \xi) \cup D(R)$ for all $n>N$. If a point is in $D(R)$ but not in $C+\delta \xi$, it must be in $F=\left(\mathrm{Cl} C^{\sim}+\delta \xi\right) \cap D(R)$. Now $F$ is a compact set and disjoint from $X$, so that $d(X)>0$ on $F$. Moreover, the convergence $d\left(X_{n}\right) \rightarrow d(X)$ is uniform on $F$, so that there exists $N^{\prime}$, which we may choose $>N$, such that $d\left(X_{n}\right)>0$ on $F$ for all $n>N^{\prime}$. This implies that $X_{n} \subset C+\delta \xi$ for all $n>N^{\prime}$. The condition $n>N^{\prime}$ will be understood in the following. Since $X_{n}$ is convex, there is $\eta_{n}$ with $\left|\eta_{n}\right|=1$ such that $X_{n} \subset H_{\eta_{n}}+\delta \xi \subset C+\delta \xi$. Then $H_{\eta_{n}} \subset C$ so 
that $\left|\eta_{n}-\xi\right|<\rho$. Furthermore, any $x \in X_{n}$ is also in $H_{\eta_{n}}+\delta \xi$, so that $\eta_{n} \cdot(x-\delta \xi) \leqq 0$, or $\eta_{n} \cdot x \leqq \delta \eta_{n} \cdot \xi \leqq \delta$. By taking the supremum over all $x \in X_{n}$ we get $h\left(X_{n}, \eta_{n}\right) \leqq \delta$. Since ${ }_{\rho} h\left(X_{n}, \xi\right) \leqq h\left(X_{n}, \eta_{n}\right)$, we have ${ }_{\rho} h\left(X_{n}, \xi\right) \leqq \delta$. After taking lim sup and observing that $\delta$ is arbitrary, the lemma is proved.

LEMMA : 5.3. If $X$ is convex, nonempty, and $0 \notin X$, then ${ }_{\rho} h(X, \xi)<0$ for any $\rho>0$ and any $\xi \neq 0$ satisfying $h(X, \xi) \leqq 0$.

Proof. We may assume in the proof that $X$ is closed and $|\xi|=1$. Let $\rho>0$ be given. The cone $C$ in the proof of Lemma 5.2 has now the property that $\mathrm{Cl} C^{\sim}$ and $X$ are closed, convex and disjoint. There is then $\delta>0$ such that $\mathrm{Cl} C^{\sim}-\delta \xi$ and $X$ are still disjoint, so that for some $\eta$ with $\eta \cdot \xi>0, X \subset H_{\eta}-\delta \xi \subset C-\delta \xi$. It follows that $H_{\eta} \subset C$ so that $|\eta-\xi|<\rho$. Every $x \in X$ is also in $H_{\eta}-\delta \xi$ so that $\eta \cdot(x+\delta \xi) \leqq 0$, or $\eta \cdot x \leqq-\delta \eta \cdot \xi<0$. Taking the supremum over all $x \in X$ we get $h(X, \eta)<0$, and therefore, since $|\eta-\xi|<\rho,{ }_{\rho} h(X, \xi)<0$.

We are now in a position to prove Theorem 5.1 below. The converse of Theorem 5.1 is also true, but we will not be able to prove it until $\$ 6$ (Theorem 6.3).

THEOREM 5.1. If $\left\{X_{n}\right\}$ is a sequence of convex sets converging to a nonempty set $X$, then $h\left(X_{n}\right) \rightarrow$ inf $h(X)$.

Proof. It suffices to show the following two inequalities for every $\xi$ with $|\xi|=1$ :

$$
\begin{aligned}
& \lim _{\rho \rightarrow 0} \lim \sup _{\rho} h\left(X_{n}, \xi\right) \leqq h(X, \xi), \\
& \lim _{\rho \rightarrow 0} \liminf \sin _{\rho} h\left(X_{n}, \xi\right) \geqq h(X, \xi) .
\end{aligned}
$$

A change of origin by an amount $a \in E^{m}$ produces a change $\xi \cdot a$ in all support functions. To show (5.2) and (5.3) we may therefore choose the origin at pleasure. For any $\xi$ such that $h(X, \xi)=\infty,(5.2)$ is automatically satisfied. If $h(X, \xi)<\infty$, make a change of origin, if necessary, so that $h(X, \xi)=0$. Then (5.2) follows from Lemma 5.2. To establish (5.3), take any $x \in X$ and choose any $\varepsilon>0$. Since $X_{n} \rightarrow X$, there exists $N$ such that for every $n>N$ there is $x_{n} \in X_{n}$ with $\left|x_{n}-x\right|<\varepsilon$. The condition $n>N$ will be understood in the following. Now for any $\eta$ we have

$$
\eta \cdot x_{n}=\xi \cdot x+\xi \cdot\left(x_{n}-x\right)+(\eta-\xi) \cdot x_{n} .
$$

If $|\eta-\xi|<\rho$, then $\left|\xi \cdot\left(x_{n}-x\right)+(\eta-\xi) \cdot x_{n}\right|<\varepsilon+\rho(|x|+\varepsilon)$, so that $\eta \cdot x_{n}>$ $\xi \cdot x-\varepsilon-\rho(|x|+\varepsilon)$. For such $\eta$ we have then $h\left(X_{n}, \eta\right) \geqq \eta \cdot x_{n}>\xi \cdot x-\varepsilon-\rho(|x|+\varepsilon)$. Taking the infimum over all $\eta$ with $|\eta-\xi|<\rho$, and then taking lim inf, we obtain $\lim \inf { }_{\rho} h\left(X_{n}, \xi\right) \geqq \xi \cdot x-\varepsilon-\rho(|x|+\varepsilon)$. Since $\varepsilon$ is arbitrary, $\lim \inf _{\rho} h\left(X_{n}, \xi\right) \geqq \xi \cdot x-\rho|x|$. Taking now first the limit as $\rho \rightarrow 0$, and then the supremum over all $x \in X$, establishes (5.3).

A simple counterexample shows that Theorem 5.1 is not valid without the convexity condition: on the real line take $X_{n}$ to consist of the two points 0 and $n$. 
6. Conjugate functions. In this section all functions $f, f_{n}, \phi, \phi_{n}$ are understood to be convex, closed and defined on $E^{m}$. We shall adopt from [3] the symbol $[X, f]$, in which $X$ is the set on which $f$ is finite, and $[X, f]=\{(x, a)$ : $x \in X, a \geqq f(x)\}$. Thus, $[X, f]$ is $a$ subset of $E^{m+1}$. $X$ is convex since $f$ is, but $X$ is not necessarily closed. However, $[X, f]$ is always closed as a result of $f$ being closed. The set $[X, f]$ can be thought of as the region above the graph of $f$.

THEOREM 6.1. $\left[X_{n}, f_{n}\right] \rightarrow[X, f]$ if and only if $f_{n} \rightarrow{ }_{\text {inf }} f$.

Proof. We shall prove the following two equivalences:

$$
\begin{aligned}
& d\left(\left[X_{n}, f_{n}\right],(x, a)\right) \rightarrow 0 \text { for all } f(x) \leqq a<\infty \\
& \Leftrightarrow \lim _{\rho \rightarrow 0} \lim \sup _{\rho} f_{n} \leqq f, \\
& \liminf d\left(\left[X_{n}, f_{n}\right],(x, a)\right)>0 \text { for all } a<f(x) \\
& \Leftrightarrow \lim _{\rho \rightarrow 0} \liminf _{\rho} f_{n} \geqq f .
\end{aligned}
$$

Note that the right-hand sides of (6.1) and (6.2) together are equivalent to $f_{n} \rightarrow$ inf $f$, and the left-hand sides together are equivalent to $\left[X_{n}, f_{n}\right] \rightarrow[X, f]$, using Theorem 3.1.

To show (6.1), assume the left-hand side. The right-hand side is trivially true for any $x$ for which $f(x)=\infty$. Assume therefore $f(x)<\infty$ and take $a=f(x)$. According to the left-hand side of (6.1) there exists a sequence $\left\{\left(x_{n}, a_{n}\right)\right\}$ with $x_{n} \in X_{n}, a_{n} \geqq f\left(x_{n}\right), x_{n} \rightarrow x, a_{n} \rightarrow f(x)$. So for any $\rho>0$ lim sup ${ }_{p} f_{n}(x)$ $\leqq \lim \sup f_{n}\left(x_{n}\right) \leqq \lim \sup a_{n}=f(x)$. Taking the limit as $\rho \rightarrow 0$ establishes the implication $\Rightarrow$. Assume now the right-hand side of (6.1) and let $f(x) \leqq a<\infty$. Since lim $\sup _{\rho} f_{n}(x)$ is nondecreasing as $\rho$ decreases, for any $\rho>0$ we have lim sup $f_{\rho}(x) \leqq \lim _{\rho \rightarrow 0}$ lim sup ${ }_{\rho} f_{n}(x) \leqq f(x)$ by the right-hand side of (6.1). Take $\rho>0$ arbitrary, then there exists a sequence $\left\{x_{n}\right\}$ with $\left|x_{n}-x\right|<\rho$ such that $\lim \sup f_{n}\left(x_{n}\right) \leqq f(x)$, which is $\leqq a$. Put $a_{n}=\max \left(f_{n}\left(x_{n}\right), a\right)$, then $\left(x_{n}, a_{n}\right) \in\left[X_{n}, f_{n}\right]$ and $a_{n} \rightarrow a$ so that $\lim \sup d\left(\left(x_{n}, a_{n}\right),(x, a)\right) \leqq \rho$, implying $\lim \sup d\left(\left[X_{n}, f_{n}\right],(x, a)\right) \leqq \rho$. Since $\rho$ is arbitrary, the left-hand side of (6.1) follows.

To show (6.2), assume the left-hand side and take any $a<f(x)$. There is $N$ and a cylindrical neighborhood about $(x, a)$ of points $(y, b)$ with $|y-x|<\rho,|b-a|<\varepsilon$, such that this neighborhood is disjoint from $\left[X_{n}, f_{n}\right]$ for all $n>N$. That is, there is $N, \rho>0$ and $\varepsilon>0$ such that ${ }_{\rho} f_{n}(x) \geqq a+\varepsilon$ for all $n>N$. We have then $\lim \inf { }_{\rho} f_{n}(x) \geqq a+\varepsilon$, so $\lim _{\rho \rightarrow 0} \lim \inf _{\rho} f_{n}(x)>a$ for all $a<f\left(x_{n}\right)$, implying the right-hand side of (6.2). Finally, assume the right-hand side of (6.2) and let $x, a$ be such that $a<f(x)$. Put $2 \varepsilon=f(x)-a$, then there exists $N$ and $\rho>0$ such that $f_{n}(x)>a+\varepsilon$ for all $n>N$. For such $n d\left(\left[X_{n}, f_{n}\right],(x, a)\right)>\min (\rho, \varepsilon)$, so that $\lim \inf d\left(\left[X_{n}, f_{n}\right],(x, a)\right) \geqq \min (\rho, \varepsilon)>0$. This concludes the proof. 
The conjugate function [3] of a not identically infinite function $f$ is the function $\phi$ on $E^{m}$ given by $\phi(\xi)=\sup _{x}(\xi \cdot x-f(x))$. Obviously, one may restrict the supremum over all $x \in X=\{x: f(x)<\infty\}$. From the definition of $\phi$ it follows that it is the support function of $[X, f]$ at $(\xi,-1) . \phi$ is closed, and the relationship of being conjugate is reciprocal [3]. The following theorem shows that infimal convergence is preserved by conjugation.

THEOREM 6.2. Let $\phi_{n}$, $\phi$ be the conjugate functions of $f_{n}$, $f$, none of the functions being identicallly $\infty$, then $f_{n} \rightarrow{ }_{\text {inf }} f$ if and only if $\phi_{n} \rightarrow{ }_{\text {inf }} \phi$. Equivalently $\left[X_{n}, f_{n}\right] \rightarrow[X, f]$ if and only if $\left[\Xi_{n}, \phi_{n}\right] \rightarrow[\Xi, \phi]$.

Proof. That the two statements are equivalent follows immediately from Theorem 6.1. We need only show $f_{n} \rightarrow$ inf $f \Rightarrow \phi_{n} \rightarrow$ inf $\phi$, since conjugation is reciprocal. According to Theorem $6.1 f_{n} \rightarrow$ inf $f$ implies $\left[X_{n}, f_{n}\right] \rightarrow[X, f]$, and this, in turn, implies $h\left(\left[X_{n}, f_{n}\right]\right) \rightarrow$ inf $h([X, f])$, by Theorem 5.1. Evaluating the support functions at $(\xi,-1)$ we have then

$$
\lim _{\rho \rightarrow 0} \lim \inf _{\rho} h\left(\left[X_{n}, f_{n}\right], \xi,-1\right)=h([X, f], \xi,-1),
$$

and similarly with lim inf replaced by lim sup. In (6.3) ${ }_{\rho} h$ of a set is defined as the infimum of $h$ taken over a spherical neighborhood of $(\xi,-1)$, i.e., all points $(\eta,-\zeta)$ with $d((\eta,-\zeta),(\xi,-1))<\rho$. Since we let $\rho \rightarrow 0$, we may instead take the infimum over cylindrical neighborhoods $\{(\eta,-\zeta):|\eta-\xi|<\rho$ and $|\zeta-1|<\rho\}$, and we shall understand ${ }_{\rho} h$ in that sense in the remainder of the proof. Now for $\zeta>0$, since support functions are positively homogeneous, we have

$$
h\left(\left[X_{n}, f_{n}\right], \eta,-\zeta\right)=\zeta h\left(\left[X_{n}, f_{n}\right], \eta / \zeta,-1\right)=\zeta \phi_{n}(\eta / \zeta),
$$

so that

$$
\begin{aligned}
& \lim _{\rho \rightarrow 0} \lim \inf _{\rho} h\left(\left[X_{n}, f_{n}\right], \xi,-1\right) \\
& \quad=\lim _{\rho \rightarrow 0} \lim \inf _{\inf }|\eta-\xi|<\rho,|\zeta-1|<\rho \zeta \phi_{n}(\eta / \zeta) \\
& =\lim _{\rho \rightarrow 0} \lim \inf \inf _{|\eta-\xi|<\rho,|\zeta-1|<\rho} \phi_{n}(\eta / \zeta) .
\end{aligned}
$$

For every $\rho>0$ there is $\varepsilon>0$ such that $|\eta-\xi|<\varepsilon$ and $|\zeta-1|<\varepsilon$ together imply $|\eta| \zeta-\xi \mid<\rho$, and $\varepsilon \rightarrow 0$ as $\rho \rightarrow 0$ (we may take $\varepsilon=\rho /(2+|\xi|)$ ). From this follows

$$
\begin{aligned}
\inf _{|\eta-\xi|<\rho,|\zeta-1|<\rho} \phi_{n}(\eta \mid \zeta) & \leqq \inf _{|\eta-\xi|<\rho} \phi_{n}(\eta) \\
& \leqq \inf _{|\eta-\xi|<\varepsilon,|\zeta-1|<\varepsilon} \phi_{n}(\eta / \zeta) .
\end{aligned}
$$

Taking $\lim _{\rho \rightarrow 0} \lim$ inf in (6.5) throughout, the extreme members are equal, so that they are equal to the middle member $\lim _{\rho \rightarrow 0} \lim \inf { }_{\rho} \phi_{n}(\xi)$. Substituting this on the right-hand side of (6.4) and replacing the left-hand side of (6.4) by the right-hand side of (6.3), which equals $\phi(\xi)$, we get $\phi(\xi)=\lim _{\rho \rightarrow 0} \lim _{\inf } \phi_{n}(\xi)$, 
and similarly with lim inf replaced by lim sup. By definition, we have then $\phi_{n} \rightarrow_{\text {inf }} \phi$, and the theorem is proved.

We are now in a position to prove the converse of Theorem 5.1.

THEOREM 6.3. If $\left\{X_{n}\right\}$ is a sequence of convex sets, $X$ a nonempty convex set, and $h\left(X_{n}\right) \rightarrow$ inf $h(X)$, then $X_{n} \rightarrow X$.

Proof. We may assume the $X_{n}$ and $X$ closed. Define $f$ by $f(x)=0$ for $x \in X$, $f(x)=\infty$ for $x \notin X$, then $f$ is convex and closed. Moreover, the conjugate function $\phi$ is identical to the support function $h(x)$. Define $f_{n}, \phi_{n}$ similarly in terms of $X_{n}$. Obviously, $X_{n} \rightarrow X$ if and only if $\left[X_{n}, f_{n}\right] \rightarrow[X, f]$. By hypothesis, $h\left(X_{n}\right) \rightarrow$ inf $h(X)$, or $\phi_{n} \rightarrow_{\text {inf }} \phi$. By Theorem 6.2 this implies $f_{n} \rightarrow$ inf $f$, and by Theorem 6.1 the latter implies $\left[X_{n}, f_{n}\right] \rightarrow[X, f]$

Q.E.D.

Second proof of Theorem 4.1. Using Theorem 6.3, it suffices to show $h\left(P\left(X_{n}\right)\right) \rightarrow{ }_{\text {inf }} h(P(X))$. Now for any nonempty set $X, h(P(X))=0$ whenever $h(X) \leqq 0$ and $h(P(X))=\infty$ whenever $h(X)>0$. Therefore, it is sufficient to show

$$
\begin{array}{ll}
\lim _{\rho \rightarrow 0} \lim \sup _{\rho} h\left(P\left(X_{n}\right), \xi\right)=0 & \text { if } h(X, \xi) \leqq 0, \\
\lim _{\rho \rightarrow 0} \lim \inf _{\rho} h\left(P\left(X_{n}\right), \xi\right)=\infty & \text { if } h(X, \xi)>0 .
\end{array}
$$

Here (6.6) is trivially true if $\xi=0$. Suppose that $\xi \neq 0$ is such that $h(X, \xi) \leqq 0$, then, using Lemma 5.3, for every $\rho>0,{ }_{\rho} h(X, \xi)<0$. Since $X_{n} \rightarrow X$ we have $h\left(X_{n}\right) \rightarrow_{\text {inf }} h(X)$ by Theorem 5.1. Thus using Lemma 5.1, we have for every $\rho>0, \lim \sup _{\rho} h\left(X_{n}, \xi\right) \leqq{ }_{\rho} h(X, \xi)$, which was shown above to be $<0$. It follows that $\lim \sup _{\rho} h\left(P\left(X_{n}\right), \xi\right)=0$. Taking the limit as $\rho \rightarrow 0$ yields (6.6). Now suppose $\xi$ is such that $h(X, \xi)>0$. Since $h\left(X_{n}\right) \rightarrow_{\text {inf }} h(X)$, we have $\lim _{\rho \rightarrow 0} \lim \inf _{\rho} h\left(X_{n}, \xi\right)=h(X, \xi)>0$, so that there exist $\rho$ and $N$ such that ${ }_{\rho} h\left(X_{n} \xi\right)>0$ for all $n>N$. For such $\rho$ and $n$ we have then ${ }_{\rho} h\left(P\left(X_{n}\right), \xi\right)=\infty$, and taking first lim inf and then the limit as $\rho \rightarrow 0$ establishes (6.7).

7. Level sets and polars. For any function $f$ and real number $a$ we define the level set of $f$ relative to $a$ as $L_{a}(f)=\{x: f(x) \leqq a\}$. The level sets of $f$ are convex if $f$ is convex, and closed if $f$ is closed. In the following, we shall write inf $f$ to mean the infimum of $f(x)$ over all $x$.

THEOREM 7.1 Given a sequence $\left\{f_{n}\right\}$ of functions and a convex function $f$ such that $f_{n} \rightarrow{ }_{\inf } f$. Then $L_{a}\left(f_{n}\right) \rightarrow L_{a}(f)$ if $a \neq \inf f$.

Proof. Note that $f$ is closed by Corollary 5.1. The conclusion of the theorem is implied by Theorem 3.1 if we show that $d\left(L_{a}\left(f_{n}\right), x\right) \rightarrow 0$ for every $x \in L_{a}(f)$ and $\lim \inf d\left(L_{a}\left(f_{n}\right), x\right)>0$ for every $x \notin L_{a}(f)$. Suppose first $x \in L_{a}(f)$, or $f(x) \leqq a$. Since we assume $a \neq \inf f$, we must have $a>\inf f$. If $f(x)>\inf f$ then given any $\rho>0$ we can always find $y$ with $|y-x|<\rho$ such that $f(y)<f(x)$, using the convexity of $f$. In that case: ${ }_{\rho} f(x)<f(x)$ so that 
${ }_{\rho} f(x)<a$, for every $\rho>0$. The latter is, of course, also true if $f(x)=\inf f$. Since $f_{n} \rightarrow$ inf $f$, by definition we have $\lim _{\rho \rightarrow 0} \lim \sup _{\rho} f_{n}=f$, and then replacing in (5.1) $f^{*}$ by $f$ we get that for every $\rho>0$, $\lim \sup _{\rho} f_{n}(x) \leqq{ }_{\rho} f(x)<a$. Thus, there exists $N$ such that ${ }_{\rho} f_{n}(x)<a$ for all $n>N$. Therefore, for each $n>N$ there exists $x_{n} \in L_{a}\left(f_{n}\right)$ with $\left|x_{n}-x\right|<\rho$. This implies $d\left(L_{a}\left(f_{n}\right), x\right)<\rho$ for $n>N$, so $\lim \sup d\left(L_{a}\left(f_{n}\right), x\right) \leqq \rho$. Since $\rho$ is arbitrary, $d\left(L_{a}\left(f_{n}\right), x\right) \rightarrow 0$. Now suppose $x \notin L_{a}(f)$, then $f(x)>a$. Since $\lim _{\rho \rightarrow 0} \liminf f_{\rho}(x)=f(x)>a$, there exists $\rho$ and $N$ such that $f_{\rho}(x)>a$ for all $n>N$. This means that for all $n>N$ and all $y$ with $|y-x|<\rho, f_{n}(y)>a$ so $y \notin L_{a}\left(f_{n}\right)$. This, in turn, implies that $d\left(L_{a}\left(f_{n}\right), x\right) \geqq \rho$ for $n>N$, so that $\lim \inf d\left(L_{a}\left(f_{n}\right), x\right) \geqq \rho>0$. This concludes the proof.

Theorem 7.1 is false if $a=\inf f$, or if infimal convergence is replaced by pointwise convergence, or if $f$ is not convex, as illustrated by the following counterexamples.

EXAMPLE 7.1. The domain of the functions is the real line; $f(x)=0$ for all $x ; f_{n}(x)=0$ for $x<0, f_{n}(x)=x / n$ for $x \geqq 0 ; a=0$. Here $a=\inf f, L_{a}(f)$ is the whole real line, but $L_{a}\left(f_{n}\right)$ is the nonpositive half line for all $n$, so $L_{a}\left(f_{n}\right) \rightarrow L_{a}(f)$ in spite of the fact that $f_{n} \rightarrow$ inf $f$.

EXAmple 7.2 (this is closely related to Example 5.2). The domain is the plane; $f(x, y)=x$ for $x \geqq 0, y=0$, and $f(x, y)=\infty$ otherwise; $f_{n}(x, y)=x+n y$ for $x \geqq 0,0 \leqq-n y \leqq x$, and $f_{n}(x, y)=\infty$ otherwise; $a=1$. Here inf $f=0<a$, $f_{n} \rightarrow f, L_{a}(f)=\{(x, y): 0 \leqq x \leqq 1, y=0\}$, whereas $L_{a}\left(f_{n}\right) \rightarrow\{(x, y): x \geqq 0, y=0\}$.

EXAMPLE 7.3. The domain is the real line; $f(x)=x$ for $x<0, f(x)=0$ for $x \geqq 0 ; f_{n}(x)=x$ for $x<1 / n, f_{n}(x)=1 / n$ for $x \geqq 1 / n ; a=0$. Here $a>\inf f$, $f_{n} \rightarrow{ }_{\text {inf }} f$, but $L_{a}(f)$ and $L_{a}\left(f_{n}\right)$ are as in Example 7.1.

The polar (o1 dual) of a nonempty set $X$ is defined as [2] $X^{*}=\{\xi: \xi \cdot x \leqq 1$ for all $x \in X\} . X^{*}$ is always convex and closed. If $X$ is convex and closed and $0 \in X$, then $X^{* *}=X$ [2]. If $C$ is a cone, its polar can also be written as $C^{*}=\{\xi: \xi \cdot x \leqq 0$ for all $x \in C\}$, since $\sup _{x \in C} \xi \cdot x$ is either 0 or $\infty$. By the definition of support function, we can write $X^{*}$ as $X^{*}=\{\xi: h(X, \xi) \leqq 1\}=L_{1}(h(X))$, i.e., the level set of $h(x)$ relative to 1 . For a cone $C$ we may replace 1 by $0: C^{*}=L_{0}(h(C))$.

THEOREM 7.2. Let $\left\{X_{n}\right\}$ be a sequence of convex sets and $X$ a nonempty set. Then $X_{n} \rightarrow X$ implies $X_{n}^{*} \rightarrow X^{*}$.

Proof. According to Theorem $5.1 h\left(X_{n}\right) \rightarrow_{\text {inf }} h(X)$. Since inf $h(X) \leqq 0$ (every support function equals 0 in $\xi=0$ ) the conclusion of the theorem follows from Theorem 7.1 after replacing $f_{n}, f$ by $h\left(X_{n}\right), h(X)$, and $a$ by 1 .

The same counterexample that was used after Theorem 5.1 shows that Theorem 7.2 is not true without the convexity condition.

Third proof of Theorem 4.1. Put $C=\operatorname{Cl} P(X), C_{n}=\operatorname{Cl} P\left(X_{n}\right)$, then it suffices to show $C_{n} \rightarrow C$. Since $C_{n}, C$ are closed, convex, and contain $0, C_{n}^{* *}=C_{n}$, $C^{* *}=C$. Therefore, it is sufficient to show $C_{n}^{* *} \rightarrow C^{* *}$. According to Theorem 
7.2, it is then sufficient to show $C_{n}^{*} \rightarrow C^{*}$, since the $C_{n}^{*}$ are convex and $C^{*}$ is nonempty. Now $\xi \cdot x \leqq 0$ if and only if $\xi \cdot(\lambda x) \leqq 0$ for all $\lambda>0$, from which it follows that $\xi \cdot x \leqq 0$ for all $x \in X$ if and only if $\xi \cdot x \leqq 0$ for all $x \in P(x)$. Hence $C^{*}=L_{0}(h(C))=L_{0}(h(P(X)))=L_{0}(h(X))$, and similarly $C_{n}^{*}=L_{0}\left(h\left(X_{n}\right)\right)$. Since $0 \notin X, 0$ and $X$ can be strictly separated by a hyperplane, and it follows that there exists $\xi$ such that $h(X, \xi)<0$. Hence $\inf h(X)<0$. By Theorem 5.1 we have $h\left(X_{n}\right) \rightarrow{ }_{\text {inf }} h(X)$, and then using Theorem 7.1 with $f_{n}=h\left(X_{n}\right), f=h(X)$, $a=0$, we get $L_{0}\left(h\left(X_{n}\right)\right) \rightarrow L_{0}(h(X))$, or $C_{n}^{*} \rightarrow C^{*}$, and the proof is complete.

\section{REFERENCES}

1. D. L. Burkholder and R. A. Wijsman, Optimum properties and admissibility of sequential tests, Ann. Math. Statist. 34 (1963), 1-17.

2. H. G. Eggleston, Convexity, Cambridge Univ. Press, London and New York, 1958.

3. W. Fenchel, Convex cones, sets, and functions, Mimeographed notes by D. W. Blackett, Princeton Univ. Press, Princeton, N. J., 1953.

4. Z. Frolík, Cocerning topological convergence of sets, Czechoslovak Math. J. 10 (1960), $168-180$.

5. H. Hadwiger, Vorlesungen über Inhalt, Oberfäche und Isoperimetrie, Springer-Verlag, Berlin, 1957.

6. R. A. Wijsman, Convergence of sequences of convex sets, cones and functions, Bull. Amer. Math. Soc. 70 (1964), 186-188.

UNIVERSITY OF ILLINOIS,

URBANA, ILLINOIS 